\title{
My mother was saved from hip fracture by treatment for osteoporosis, but will I be?-Implications on risk estimates from successful osteoporosis treatment
}

\author{
B. E. Rosengren • M. K. Karlsson
}

Received: 24 May 2012 / Accepted: 30 May 2012 / Published online: 16 June 2012

(C) International Osteoporosis Foundation and National Osteoporosis Foundation 2012

Recently, the question of the validity of FRAX measurements [1] in individuals treated with osteoporosis pharmacotherapy has been discussed [2]. I would like to highlight the theoretical impact of the fracture protective therapies introduced and widely used in the recent 15 years in terms of current fracture risk estimates for the offspring of the treated individuals.

In a theoretical 60-year old Swedish woman $165 \mathrm{~cm}$, $70 \mathrm{~kg}$ without any other risk factors the FRAX 10 year probability for major osteoporotic fracture is $7.3 \%$ and for hip fracture $1.1 \%$. However, with a parent hip fracture, the probabilities rise to 14 and $1.5 \%$. Anti-osteoporotic treatment in postmenopausal women with bisphosphonates reduces hip fracture risk with approximately $40 \%$ in RCTs [3] and has been used for almost 15 years in Sweden. Many hip fractures have been avoided resulting in too conservative FRAX probabilities for the offspring of the individuals in which a hip fracture was avoided by pharmacotherapy.

With this in mind, it might be interesting to explore the possibility to include parental treatment for osteoporosis in the algorithm. Apart from addressing the described problem, this would also be of interest as the genetic predisposition for osteoporosis would be accounted for, maybe most interesting for FRAX estimates without DXA measurements.

Conflicts of interest None.

\section{References}

1. De Laet C, Oden A, Johansson H, Johnell O, Jonsson B, Kanis JA (2005) The impact of the use of multiple risk indicators for fracture on case-finding strategies: a mathematical approach. Osteoporos Int 16(3):313-318. doi:10.1007/s00198-004-1689-z

2. Leslie WD, Lix LM, Johansson H, Oden A, McCloskey E, Kanis JA Does osteoporosis therapy invalidate FRAX for fracture prediction? J Bone Miner Res. doi:10.1002/jbmr.1582

3. Bilezikian JP (2009) Efficacy of bisphosphonates in reducing fracture risk in postmenopausal osteoporosis. Am J Med 122(2 Suppl): S14-21. doi:10.1016/j.amjmed.2008.12.003
B. E. Rosengren $(\bowtie) \cdot$ M. K. Karlsson

Clinical and Molecular Osteoporosis Research Unit,

Department of Orthopedics, Clinical Sciences Malmö,

Lund University,

Malmö, Sweden

e-mail: bjorn.rosengren@med.lu.se 